\title{
CALCULATIONAL ESTIMATIONS OF NEUTRON YIELD FROM ADS TARGET
}

\author{
I.I. Degtyarev, O.A. Liashenko, I.A.Yazynin, IHEP,142284 Protvino, Russia; \\ V.I. Belyakov-Bodin, ITEP,117259 Moscow, Russia; \\ A.I. Blokhin, IPPE, 249020 Obninsk, Russia .
}

\begin{abstract}
Results of computational studies of high power spallation thick ADS (Accelerator-Driven System) targets with 0.8$1.2 \mathrm{GeV}$ proton beams are given. Comparisons of experiments and calculations of double differential and integral $\mathrm{n} / \mathrm{p}$ yield are also described.
\end{abstract}

\section{PARTICLE PRODUCTION MODELS}

\subsection{Theory driven model (Intermediate energy).}

The intra-nuclear cascade-exciton model is one of the most popular to simulate of the secondary particle parameters for the $h(\gamma)$ A-inelastic scattering in the intermediate region and traditionally applied to reaction above $\sim 100 \mathrm{MeV}$. It allows one to reproduce the experimental observations up to energies $\sim 10-20 \mathrm{GeV}$. We have developed a new model version (CEM-RTS\&T) and included it as event generator to the RTS\&T code [1]. In the CEM-RTS\&T model the $h(\gamma)$-induced nuclear reaction is assumed to be three-step process: intranuclear cascade stage (INC), pre-equilibrium decay of residual nucleus and the compound nucleus decay process (evaporation/fission competition). In the INC model nuclear structure is treated as a two-component degenerated Fermi-gas of nucleons in the spherical type of nuclear density The phenomenological potential for nucleons is described as sum of the nuclear and Coulomb terms: $V^{N}(r)=V_{N}^{N}(r)+V_{C}(r), \quad$ here $V_{N}^{N}(r)=T^{F}(r)+B(A, Z)$, where Fermi-energy depends on the local density of the nucleus $\rho(r): \quad T^{F}(r)=\frac{\hbar}{2 m}\left[3 \pi^{2} \rho(r)\right]^{1 / 3}$. The nucleon distribution density is of fundamental parameter of INC model. The most popular Woods-Saxon form does not satisfy the two physical requirements, namely, the asymptotic behavior and the behavior near the center of the nucleus. The semi-phenomenological nucleon density distribution proposed by Gambhir and Patil [2] in the form:

$$
\rho_{i}(r)=\rho_{o i}^{N}\left\{1+\left[\frac{1}{2}+\frac{1}{2}\left(\frac{r}{R}\right)^{2}\right]^{\alpha_{i}}\left[e^{\frac{r-R}{a_{i}}}+e^{-\frac{r+R}{a_{i}}}\right]\right\}^{-1},
$$

where $R$ is a measured size of nucleus and $a_{i}$ and $\quad \alpha_{i}$ are given in terms of the separation energy $S_{i}$ of the last nucleon through: $a_{i}=\frac{\hbar}{2 \sqrt{2 m S_{i}}} ; \alpha_{i}=\frac{q}{\hbar} \sqrt{\frac{m}{2 S_{i}}}+1$. Here $m$ is the nucleon rest mass, $q=0(i=n)$ and $q=Z-1(i=p)$. This algebraic form designed has been tested in detail by comparing with the experiment the form factors for the electron scattering. The Coulomb potential (only for protons) $V_{c}(r)=\left(Z e^{2} / 2 R_{c}\right)\left[3-\left(r / R_{c}\right)^{2}\right] \quad\left(r \leq R_{c}\right) \quad$ and $V_{c}(r)=Z e^{2} / r\left(r>R_{c}\right)$, where $R_{C}$ is the Coulomb radius. The $\pi(K) A$-interaction potential is taken as $V^{\pi(K)}(r)=-V_{0}^{\pi(K)} \Theta(r), \quad$ where $\quad V_{0}^{\pi}=25 \mathrm{MeV}$, $V_{0}^{K}=30 \mathrm{MeV}, \Theta(r)-$ is the unit step function. The center-mass correlations are taken into account as proposed in [3]. One-particle nuclear density $\widetilde{\rho}(\vec{r})$ taken from the equation:

$$
\rho(\vec{r})=\int\left[\prod_{i=1}^{A} \tilde{\rho}\left(\vec{r}_{i}\right)\right] \delta\left(\sum_{i=1}^{A} \frac{\vec{r}_{i}}{A}\right) \prod_{i=2}^{A} d^{3} \vec{r}_{i}
$$

where $\vec{r}_{i}$-is the coordinates of $\mathrm{i}$-th nucleon. The Pauli blocking is incorporated in the cascade simulation procedure. The effects of particle refraction by nuclear potential and the decrease of nucleon density during intranuclear cascade (co-called trailing effect) are taken into account also. The integral $h h$ and $\not h$ cross sections taken from the Particle Data Group compilation. Assuming the charge symmetry the following channel cross sections are taken identical:

$$
n n=p p, \pi^{-} p=\pi^{+} n, \pi^{-} n=\pi^{+} p, K^{0} p=K^{+} n ; K^{0} n=K^{+} p .
$$

The isobar production channels covered by the INC model are presented in Table 1. The strange channels like $\not p \rightarrow \Sigma K^{+}\left(\Lambda K^{+}, \Lambda K^{0} \pi^{+}\right)$and non-strange pseudo-scalar and vector meson photoproduction channels $\quad \gamma N \rightarrow(\rho / \omega / \eta) N$ are included also. For multi-particle final states we adopt the CERN library GENBOD (W515) routine.

Table 1: Baryon resonance production channels.

\begin{tabular}{|c|c|}
\hline Entrance channel & Exit channel \\
\hline$p p$ & $p \Delta^{+}, n \Delta^{++}, \Delta^{+} \Delta^{+}, \Delta^{0} \Delta^{++}$ \\
\hline$p n$ & $n \Delta^{+}, p \Delta^{0}, \Delta^{0} \Delta^{+}, \Delta^{-} \Delta^{++}$ \\
\hline$\not p$ & $\Delta^{++} \pi^{-}, \Delta^{++} \pi^{0}, \Delta^{0} \pi^{+}$ \\
\hline$p n$ & $\Delta^{+} \pi^{-}, \Delta^{0} \pi^{0}, \Delta^{-} \pi^{+}$ \\
\hline
\end{tabular}


The pre-equilibrium stage of nuclear reaction simulation is based on the exciton model. The initial exciton configuration for pre-equilibrium decay formed in cascade stage of reaction or postulated in general input ( $2 \mathrm{p} 1 \mathrm{~h}$ configuration for incoming particle or $1 \mathrm{p} 0 \mathrm{~h}$ for incoming photon). The equilibrium stage of reaction (evaporation/fission processes) is performed according to the Weisskopf-Ewing statistical theory of particle emission and Bohr and Wheeler theory of fission. Double-humped fission barrier parameters for $Z>90$ taken from the data set obtained in Obninsk and recommended by IAEA Reference Input Parameter Library (RIPL). Experimental single-humped fission barrier heights are incorporated in the RTS\&T as proposed in, or according to any theoretical models. The ratio of neutron emission to fission widths taken in form proposed by Kupriyanov et al.:

$$
\Gamma_{n} / \Gamma_{f}=\left(\widetilde{\Gamma}_{n} / \widetilde{\Gamma}_{f}\right) e^{-\frac{\delta W_{g}^{N-1}}{\widetilde{T}_{n}} e^{-\lambda U} n+\frac{\delta W_{f}}{\widetilde{T}_{f}} e^{-\lambda U f}},
$$

where $\tilde{\Gamma}_{n} / \widetilde{\Gamma}_{f}$ is Vandenbosch and Huizenga form of Fermi-gas model (asymptotic value occurring at high energies), $\delta \mathrm{W}$ is the shell correction term, $\lambda=0.05 \mathrm{MeV}$. The post-fission parameters of the fragments taken from systematics by Adeev $\left(Z_{i}, A_{i}\right)$ and Zhao et al. $\left(T_{k i n}\right)$. The inverse reaction cross section is calculated in form $\sigma_{\text {inv }}(T)=\sigma_{\text {geom }} T_{b}(T)$ with $\sigma_{\text {geom }}=\pi\left[R+R_{b}+\lambda\right]^{2}$ where $\sigma_{\text {geom }}, R, R_{b}, \lambda$ and $T_{b}(T)$ are the geometric cross section, radii of the potential residual nucleus and particle, the non-relativistic reduced channel wavelength and the non-relativistic s-wave Coulomb barrier transmission probability, respectively. The s-wave transmission factor is given by expression $T_{b}(\rho, \eta)=\left[F_{0}(\rho, \eta)^{2}+G_{0}(\rho, \eta)^{2}\right]^{-2}$, where $F_{0}, G_{0}$ are the non-relativistic zero-order regular and irregular Coulomb wave-functions of variables $\rho=\hbar^{-1}\left(R_{1}+R_{2}\right)\left(2 \mu T_{c m s}\right)^{1 / 2}$, $\eta=\hbar^{-1} Z_{1} Z_{2} e^{2}\left(\mu / 2 T_{c m s}\right)^{1 / 2}$, where $Z_{1}, Z_{2}, R_{1}, R_{2}$ are the charge and radii of two particles, respectively, $\mu$ is the reduced mass of the two particle system, $T_{c m s}$ is the kinetic energy in cms. In the current version of RTS\&T code the Fermi breakup model for disintegrating of light nuclei has replaced the evaporation model for nuclei with a mass number between 2 to 16 . The evaporation of fragments with a mass number A $>4$ does not included in current version RTS\&T. The RIPL recommended Audi and Wapstra experimental compilation of atomic masses and binding energies is used in the RTS\&T model. Level density parameter values are chosen according to the RIPL-systematics for any level density models: GilbertCameron, backed-shifted Fermi-gas model, Ignatyuk form of Fermi-gas model, generalized superfluid model (GSM), microscopic GSM, shell depended model proposed by Kataria and Ramamurthy, Mughabghab and Dunford systematic determined from the neutron resonance data. To estimate of the averaged squared matrix element two different models can be used: estimation in approximation of quasi-free scattering of a nucleon above the Fermi level on a nucleon of the target nucleus [4] or by using a set of semi-empirical parametrizations. CEM-RTS\&T has three different models to simulate the gamma-ray emission in preequilibrium and equilibrium stages: the Weisskopf singleparticle model, the Brink-Axel GDR model, and the Kopecky-Uhl generalized Lorentzian model. To calculate the partial level densities for pre-equilibrium emission simulation the Avrigeanu systematic is used. Composite formulas include the advanced pairing and shell correction in addition to the Pauli blocking effect, and average energy-depended single-particle level densities for the excited particles and holes.

\subsection{Parametrization driven model}

The existing rigorous theoretical approaches and models allow to estimate of various characteristics of secondary particle emission due to nuclear inelastic interactions, but they have uncertainly in choice of free parameters and the calculations are time-consuming. In addition, analytical representation of double differential cross sections is important to local estimation of particle fluxes in the transport simulation algorithm. On the basis of EXFOR data sets of experimental data we obtained the improved parameters of Sychev's D2N2 systematic. They represent a better statistical average, as they are based on more experimental data. The intra-nuclear cascade-exciton model is employed to calculate the cross sections which not measured. For the spallation reaction, the PDM model is applied in a form of summation of the double differential cross sections for secondary particle emission expressed in the lab. frame by $\left(\frac{d^{2} \sigma}{d T d \Omega}\right)_{i j}=\sum_{k=1}^{7}\left(\frac{d^{2} \sigma}{d T d \Omega}\right)_{k}$, where $\mathrm{i}, \mathrm{j}$ are the indexes of incident $\left(\gamma, \mathrm{N}, \pi^{ \pm}, \mathrm{K}^{ \pm}, \mathrm{K}^{0}, \bar{N}\right.$, ions $)$ and secondary $(\gamma, \mathrm{N}$, $\pi^{ \pm}, \mathrm{K}^{ \pm}, \mathrm{K}^{0}, \bar{N}$, ions, $\left.\Delta, \rho, \omega\right)$ particles. Components of $\mathrm{k}=1$ to 7 correspond to the quasi-elastic and chargeexchange scattering, lider particle emission, production of cascade particles, the pre-equilibrium/equilibrium relaxation, the fragmentation, the binary fission, and parametrized in functional form:

$\left(\frac{d^{2} \sigma}{d T d \Omega}\right)_{k}=\sigma_{i n} n_{i j} \frac{d N}{d T} f(T, \theta)$, where $\quad \sigma_{i n}, n_{i j}, f(T, \theta)$ are inelastic cross section, average multiplicity of secondary particles of $\mathrm{j}$-th type, energy-angle correlation function, respectively.

\subsection{High-energy hadronic interactions}

Simulation of $h A$ - inelastic collisions at $\mathrm{E} \geq 5-10 \mathrm{GeV}$ is based on a set of the inclusive semi-empirical formulas for one-particle production in $p p$-interactions, coupled with the additive quark model of $h A$-interactions for fast- and phenomenological model for slow particles. To 
correct simulation of the correlations in inelastic hh- and hA-interactions modified [5] FRITIOF code can be used.

\subsection{Low-energy $n$ ( $\gamma)$ A-interactions}

Transport and discrete interactions of nucleons modelling in the energy range from thermal energy up to $150 \mathrm{MeV}$ in the RTS\&T code is based on the direct uses of ENDF/B-VI evaluated data library to detailed description of low-energy interactions. Universal data reading and preparation procedure allows to use another database written in ENDF format ( JENDL, FENDL, CENDL, JEF, BROND, LA150, IAEA Photonuclear Data Library etc.). During to execution the linearization, restoration of

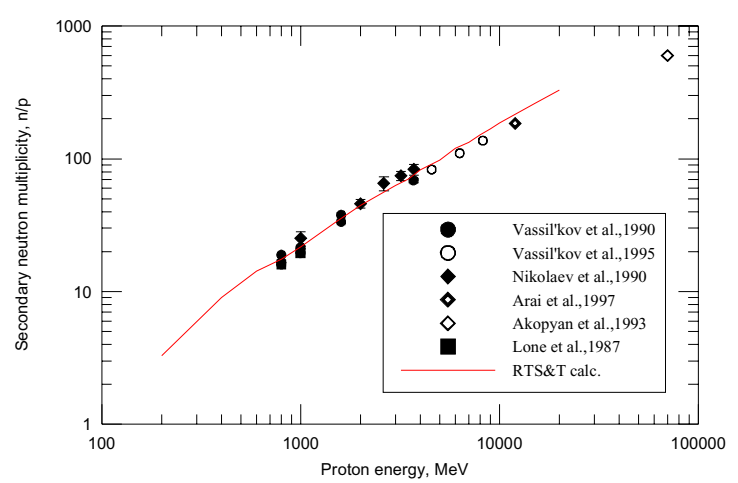

Figure 1: Total neutron yield as a function of incident proton energy.

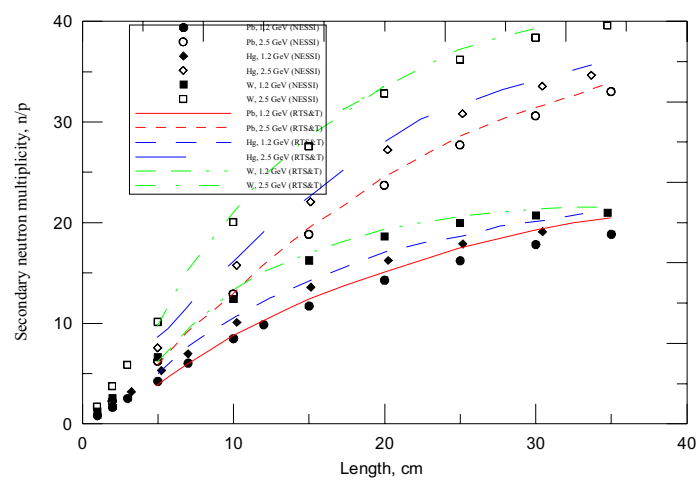

Figure 2: Total neutron yield as a function of target length.

the resolved resonances, temperature dependent Doppler broadening of the neutron cross sections and checking/correcting of angular distributions and Legendre coefficients for negative values are produced automatically with a help of Cullen's ENDF preprocessing codes LINEAR, RECENT (RECEN-DD for Reich-Moore parameters of several isotopes of JENDL library only), SIGMA1 and LEGEND [6]. Recommended interpolation schemes are used to minimize the amount of data. For data storage in memory and their further use the dynamically allocated tree of objects is organized. All types of reactions provided by ENDF-VI format are taken into account due to the nucleon transport modelling: elastic scattering, radiative capture and production of one neutron in the exit channel, absorption with production of other type particles (with division on excited states of the residual nucleus), the fission with separate yields of prompt and delayed neutrons and residual nucleus simulation by $\mathrm{MF}=8$ data, etc. The energies and angles of emitted particles are simulated according to the distributions from $M F=4,5,6,12,13,14$ and 15 files. For example, the following representations of outgoing energy-angle distributions for secondary particle can be used: tabular energy distributions, angular distributions via equally-probable cosine bins, Kalbach-Mann systematics for continuum energy-angle distributions (44 ENDF law), discrete two-body scattering, N-body phasespace energy distributions.

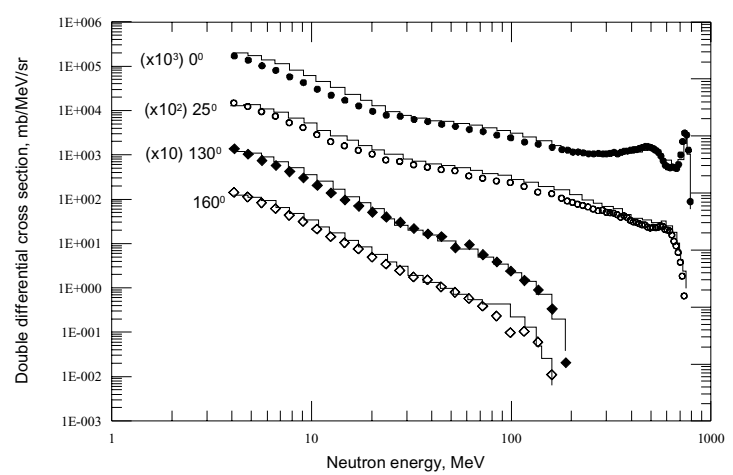

Figure 3: $P b(p, x n) X$ double differential cross sections at $E_{p}=0.8 \mathrm{GeV}$.

\section{EXPERIMENTAL DATA ANALYSIS}

The experimental results (NESSI, SATURNE) are compared with calculations performed with a RTS\&T general-purpose transport code. Comparison of total neutron yield from cylindrical lead-target $(\mathrm{d}=20 \mathrm{~cm}, \mathrm{~L}=60$ $\mathrm{cm}$ ) as a function of incident proton energy is presented in Fig. 1. Fig. 2 displays the total neutron yield from $\mathrm{Hg}$-, $\mathrm{Pb}$-, and W-cylindrical targets $(\mathrm{d}=15 \mathrm{~cm})$ irradiated by 1.2 and $2.5 \mathrm{GeV}$ proton beam. The double differential cross sections of neutron production measured at $\mathrm{Ep}=0.8 \mathrm{GeV}$ on a $2 \mathrm{~cm}$ thick $\mathrm{Pb}$ cylindrical target presented on Fig. 3 . Experimental data were obtained at the SATURNE accelerator at close angles $(0,25,130$, and 160 deg.).

\section{REFERENCES}

[1] A.I.Blokhin, I.I.Degtyarev et al., Proc. of the SARE-3 Workshop, KEK, 1997 // I.I. Degtyarev et al., IAEA Report INDC(CCP)-426, Vienna, 2001, p.161, p.171.

[2]. Y.K. Gambhir, S.H. Patil, Z.Phys. A321 (1985) 161.

[3] V.V. Uzhinskii, S.Yu. Shmakov, Yad. Fiz. 57 No. 8, pp.1532-1533

[4] K.K. Gudima et al., Nucl. Phys. A401 (1983) 329

[5] V.V.Uzhinskii, JINR preprint E2-96-192 (1996). 